\title{
AVALIAÇÃO DO SOFTWARE BIOESTAT PARA O ENSINO DE ESTATÍSTICA NOS CURSOS DE GRADUAÇÃO
}

\author{
Antônio Carlos Silveiro da SILVA ${ }^{1}$ \\ Jessica Borges da VEIGA ${ }^{2}$ \\ Bruna Borges da VEIGA ${ }^{3}$ \\ Charles CAIONI ${ }^{1}$ \\ Ademilso Sampaio de OLIVEIRA ${ }^{4}$
}

\begin{abstract}
${ }^{1}$ Engenheiro Florestal, Mestrando do Programa de Pós-Graduação Strictu Senso em Biodiversidade e Agroecossistemas Amazônicos, Universidade do Estado de Mato Grosso, Alta Floresta-MT. acsilveiro@gmail.com

${ }^{2}$ Bióloga, Mestranda do Programa de Pós-Graduação Strictu Senso em Biodiversidade e Agroecossistemas Amazônicos, Universidade do Estado de Mato Grosso, Alta Floresta-MT. jessica_mt23@ hotmail.com

${ }^{3}$ Acadêmica do curso de Graduação de Licenciatura em Matemática, Universidade do Estado de Mato Grosso, CáceresMT.bruna_nmv@hotmail.com

${ }^{4}$ Geografo, Professor Mestre do curso de Pedagogia e Administração da Faculdade de Alta Floresta, Alta Floresta MT.ademilsosampaio@hotmail.com
\end{abstract}

\section{Recebido em: 30/05/2014 - Aprovado em: 15/09/2014 - Disponibilizado em: 15/12/2014}

\begin{abstract}
RESUMO - É preciso experimentar e avaliar métodos de ensino adaptados á natureza específica da Estatística. Atualmente com advento das tecnologias computacionais o trabalho com números tem se tornado menos onerosos, com a utilização de softwares específicos. Estes vêm ganhando espaço no ambiente acadêmico, no entanto, é imprescindível a avaliação da qualidade pedagógica desta nova ferramenta educacional. Neste sentido, teve-se como objetivo demonstrar a importância da utilização do software BioEstat5.3 no ensino de estatística nos cursos de graduação, enfatizando sua facilidade e aplicabilidade em diversos testes estatísticos pelos alunos. Foi avaliada a qualidade externa do programa seguindo-se o método de check-list, verificando as características pedagógicas, facilidade de uso, características da interfase, funcionalidade, documentação e portabilidade de forma geral e específica para alguns testes estatísticos paramétricos, não-paramétricos e descritivos. Para todas as características avaliadas o programa apresentou um conceito satisfatório, principalmente pela praticidade de manuseio, fácil aquisição (gratuito e virtual) e diversidade de pacotes estatísticos. Os testes realizados obtiveram excelente desempenho,como velocidade de execução, apresentação dos resultados e facilidade de interpretação e aplicação. Portanto, o software BioEstat apresentou grande potencialidade de despertar a atenção e o interesse do aluno para com a Estatística, podendo ser amplamente utilizado para o ensino-aprendizagem nesta disciplina.
\end{abstract}

Palavras-chave - Ensino superior. Ensino-Aprendizagem. Software Livre. Programa estatístico. Qualidade de softwares.

ABSTRACT -It takes experience and evaluate teaching methods adapted to the specific nature of Statistics. Currently advent of computer technology work with numbers has become less costly, with the use of specific software. These have been gaining ground in the academic environment, however, it is essential to assess the educational quality of this new educational tool. In this sense, we aimed to demonstrate the importance of using the BioEstat 5.3 software on statistical learning in undergraduate courses, emphasizing its ease and applicability in various statistical tests by students. The external quality of the program was evaluated following the method of check-list, checking pedagogical features, ease of use, features the interphase, functionality, portability and documentation generally and specifically for some parametric tests, non-parametric and descriptive. For all traits the program showed a satisfactory concept, especially for convenience of handling, easy acquisition (Free Virtual) and diversity of statistical packages. The tests had excellent performance such as speed of execution, presentation of results and ease of interpretation and application. Therefore, the software BioEstat showed great potential to arouse attention and interest to the student with stats and can be widely used for teaching and learning of this subject .

Keywords- Higher education. Teaching and Learning. Free Software. Statistical program.Software quality.

\section{Introdução}

O que falta hoje na área da educação

são metodologias diferenciadas que

contribuam para o aprendizado do aluno. Os

softwares estão sendo aplicados para que entre outras coisas sejam um facilitador do aprendizado além de levar o aluno a se tornar conhecedor das tecnologias, pois assim, ele passa a ter acesso a esses novos meios tecnológicos. 
Segundo Paulo Freire (1996, p. 39) o aperfeiçoamento constante é essencial, pois “[...] na formação permanente dos professores, o momento fundamental é o da reflexão crítica sobre a prática. É pensando criticamente a prática de hoje e de ontem que se pode melhorar e a próxima prática".

Algo notório é que uma metodologia diferenciada quando bem aplicada possibilitam uma melhor aprendizagem, principalmente nos cursos de Estatística. Visto que,"o que se assiste, em grande parte dos cursos universitários, é uma aversão generalizada dos alunos pela disciplina de Estatística, geralmente oferecida nos primeiros semestres da graduação, e que apresenta um alto índice de reprovação" (CORDANI, 2001, p. 19-20).

Desta maneira, a inserção de softwares como auxílio no ensino-aprendizagem de Estatística nas universidades, pode se tornar uma ferramenta que possibilite o interesse e maior interação dos alunos com os conteúdos estatísticos, proporcionando uma ruptura deste paradigma estabelecido na sociedade acadêmica de aversão as disciplinas de cálculos. No entanto, "o desafio é colocar todo o potencial dessa tecnologia a serviço do aperfeiçoamento do processo educacional...’Milani (2001, p. 75).
Hoje no mercado tecnológico, é possível encontrar disponíveis, gratuitamente, diversos softwares estatísticos.Porém, é necessário verificar a qualidade destes programas no seguimento educacional. Diante do exposto, este trabalho teve como objetivo demonstrar a importância da utilização do software BioEstat no ensino de estatística nos cursos de graduação, enfatizando sua facilidade e aplicabilidade em diversos testes estatísticos pelos alunos.

\section{Materiais e Métodos}

Para a realização da pesquisa foi avaliada a qualidade do software BioEstat 5.3 (AYRES, et al., 2007). De acordo com a norma ISO/IEC 9126:1991, existem duas classificações para avaliação da qualidade desoftwares, externa e interna. A qualidade externa é visível aos usuários do sistema; qualidade interna é aquela pertinente aos desenvolvedores. Neste sentido, no presente trabalho será realizada a avaliação, apenas, da qualidade externa do produto, este tipo de procedimento avalia o programa ao longo do processo de desenvolvimento de suas funções e suas atribuições estão evidenciadas na Tabela 1.

Tabela 01 - Heurísticas pedagógicas e de usabilidade a ser verificada na análise do software BioEstat 5.3.

\begin{tabular}{ll}
\hline Heurística & Descrição \\
\hline Características pedagógicas & $\begin{array}{l}\text { Conjunto de atributos que evidenciam a conveniência e a } \\
\text { viabilidade de utilização do software no ensino de estatística. }\end{array}$ \\
\hline Facilidade de uso & Conjunto de atributos que evidenciam a facilidade de uso do \\
\hline \multicolumn{2}{c}{376} \\
Revista da Universidade Vale do Rio Verde, Três Corações, v. 12, n. 2, p. 375-385, ago./dez. 2014
\end{tabular}


software por alunos de graduação.

Características da interface

Conjunto de atributos que evidenciam a existência de meios e recursos que facilitam a interação do usuário com o software.

Funcionalidade

Conjunto de atributos que evidenciam a capacidade do software se adaptar as necessidades e preferências do usuário.

Conjunto de atributos que evidenciam que a documentação para

Documentação instalação e uso do software deve ser completa, consistente, legível e organizada.

Portabilidade Conjunto de atributos que evidenciam a adequação do software

Fonte: Adaptado de Campos e Campos (2001). aos programas disponíveis no computador.

Para elencar sua aplicabilidade utilizouse alguns testes de análises de variância paramétricose não-paramétricos, assim como de estatística descritiva. Foram analisados os seguintes parâmetros no modelo de check-list: dinâmica de realização das etapas de cada teste e a linguagem do software apresentada durante a realização destas fases assim como a apresentação de seus resultados.

\section{RESULTADOS E DISCUSSÃO}

\section{BioEstat 5.3}

O software BioEstat é um programa estatístico desenvolvido especialmente aos estudantes de graduação e pós-graduação das áreas médicas e biológicas. Seu pacote estatístico tem como objetivo propiciar aos acadêmicos de diversas áreas do conhecimento um instrumento de grande praticidade e de fácil manuseio na avaliação de informações originadas através de pesquisa. De acordo com Ayres et al. (2007), o BioEstat solidifica-se como ferramenta didática quase obrigatória devida, sobretudo, à facilidade de sua aplicação e interpretação dos fenômenos biológicos, sejam de ordem observacional, quer de natureza experimental.

Nesta perspectiva, suas características básicas estão evidenciadas na Tabela 2. O software apresentou grande potencial de uso devido à facilidade de aquisição, por meio gratuito e virtual, além de apresentar fácil instalação e possuir um manual autoexplicativo. Estas características podem conferir ao BioEstat uma alta demanda de aquisição e utilização.

Tabela 2 - Características básicas do software BioEstat 5.3.

\begin{tabular}{l|l}
\hline \multicolumn{2}{c}{ Aquisição } \\
\hline Forma & Gratuita \\
\hline Local & $\begin{array}{l}\text { http://www.mamiraua.org.br/pt- } \\
\text { br/downloads/programas/ }\end{array}$ \\
\hline Idioma & Português \\
\hline \multicolumn{2}{c}{ Portabilidade e Adaptabilidade } \\
\hline Instalação & Fácil e rápido \\
\hline $\begin{array}{l}\text { Compatível } \\
\text { com as } \\
\text { extensões do } \\
\text { computador }\end{array}$ & Sim \\
\hline $\begin{array}{l}\text { Facilidade de } \\
\text { entrada de } \\
\text { dados }\end{array}$ & Ótima \\
\hline \multicolumn{2}{c}{ Documentação } \\
\hline Possui manual & Sim, com opção Help \\
\hline $\begin{array}{l}\text { Forma de } \\
\text { aquisição }\end{array}$ & $\begin{array}{l}\text { Juntamente } \\
\text { instalação do software }\end{array}$ \\
\hline $\begin{array}{l}\text { Fácil a } \\
\text { entendimento }\end{array}$ & Sim \\
\hline
\end{tabular}




\begin{tabular}{l|ll}
\hline $\begin{array}{l}\text { Veracidade } \\
\text { das } \\
\text { informações } \\
\text { apresentada }\end{array}$ & Sim \\
\hline \multicolumn{3}{|c}{ Funcionalidade } \\
\hline $\begin{array}{l}\text { Área de } \\
\text { atuação }\end{array}$ & $\begin{array}{l}\text { Ciências } \\
\text { médicas }\end{array}$ \\
\hline $\begin{array}{l}\text { Quantidade de } \\
\text { aplicativos }\end{array}$ & 210 \\
\hline \multicolumn{2}{l}{ Fonte: Autores (2014). } \\
\hline
\end{tabular}

Oemprego do software BioEstat em sala de aula torna-se um instrumento para o ensino de estatística, o qual os alunos além de aprenderem calcular manualmente poderão desenvolver os mesmos no programa. Este novo conhecimento tornará prático e ágil o trabalho com dados, proveniente de pesquisas.Entretanto, é imprescindível que esta nova ferramenta de ensino e pesquisa seja de fácil manuseou e siga alguns conceitos de qualidade. De acordo com a norma ISO, "qualidade é a totalidade das características de um produto ou serviço que lhe confere a capacidade de satisfazer as necessidades implícitas de seus usuários"(ISO/CD8402, 1990). As características avaliadas para verificar a qualidade do programa estão evidenciadas na Tabela 3.

O software apresentou grande potencial de uso no ensino de estatística, devido sua facilidade, aplicabilidade e adaptação aos sistemas computacional.Para Campos e Campos (2001) o desenvolvimento de software educacional busca contemplar as características da educação que levam à formação global do aluno que necessita, aprender a aprender e a pensar, para melhor intervir, inovar e questionar, trabalhando desta forma com as funções da cognição.

Suas características pedagógicas apresentaramconceito ótimo, podendo ser facilmente manuseado por um acadêmico sem a presença de um instrutor, apenas com a utilização de seu manual ou com a memorização de seus processos. Akhraset al. (1995) menciona que estas características de um software educacional proporciona um ambiente de aprendizagem construtivistas.Nestes ambientes interativos a ênfase está na autonomia do aluno que interage com o ambiente, que, por sua vez, tem o foco no processo de construção do conhecimento e não apenas num domínio prédefinido do conhecimento a ser adquirido (AKHRASet al., 1995 apud CAMPOS e CAMPOS, 2001).Neste contexto, apresentase a importância da utilização de softwares na educação como forma de estímulo ao aprendizado.

Em suas características de interfase o programa obteve bom desempenho, apresentando uma linguagem simples, clareza nos procedimentos, facilidade de condução e indicando correção do conteúdo,mostrando-se satisfatórias, principalmente, por apresentar nacionalidade brasileira e conformidade com o idioma português. Isto faz com que os acadêmicos consigam desenvolver uma melhor compreensão dos procedimentos e resultados exposto pelo software. 
Tabela 3 - Resultados das características avaliadas para a qualidade externa do software BioEstat 5.3.

\begin{tabular}{|c|c|}
\hline \multicolumn{2}{|c|}{ Características pedagógicas } \\
\hline Facilidade de uso & Sim \\
\hline $\begin{array}{l}\text { Facilidade de } \\
\text { memorização }\end{array}$ & Sim \\
\hline $\begin{array}{l}\text { Grau de compreensão sem } \\
\text { a presença de um instrutor }\end{array}$ & Ótimo \\
\hline $\begin{array}{l}\text { Clareza na exposição de } \\
\text { informações }\end{array}$ & Ótima \\
\hline \multicolumn{2}{|c|}{ Características da interfase } \\
\hline $\begin{array}{l}\text { Coesão da linguagem e } \\
\text { gramática }\end{array}$ & Boa \\
\hline $\begin{array}{l}\text { Sequência lógica na } \\
\text { apresentação das fases }\end{array}$ & Ótima \\
\hline $\begin{array}{l}\text { Clareza das alternativas } \\
\text { possíveis de comando }\end{array}$ & Boa \\
\hline $\begin{array}{l}\text { Facilidade de condução } \\
\text { do programa }\end{array}$ & Ótima \\
\hline $\begin{array}{l}\text { Gestão de erro de } \\
\text { funcionamento do sistema }\end{array}$ & Ótima \\
\hline
\end{tabular}

Fonte: Autores (2014).

O programaBioEstat apresenta uma área de trabalho bem ampla e didática (Figura 1), possuindo uma linguagem de fácil compreensão e com suas ferramentas dispostas de maneira simples, possibilitando sua aprendizagem mais rápida e eficiente. Apresenta seus resultados em editor do próprio programa de forma breve e clara, podendo o usuário imprimir ou salvar os mesmos. Desenvolve testes na área de estatística básica e experimental, sendo uma excelente ferramenta para pesquisadores nas mais diversas áreas do conhecimento, principalmente biológicas, agrárias e medicas. A qualidade no desenvolvimento de alguns aplicativos disponíveis pelo programa BioEstat 5.3 estão evidencias nos tópicos seguintes.
Figura 1 - Interface da área inicial do software BioEstat 5.3 e seus comandos principais.

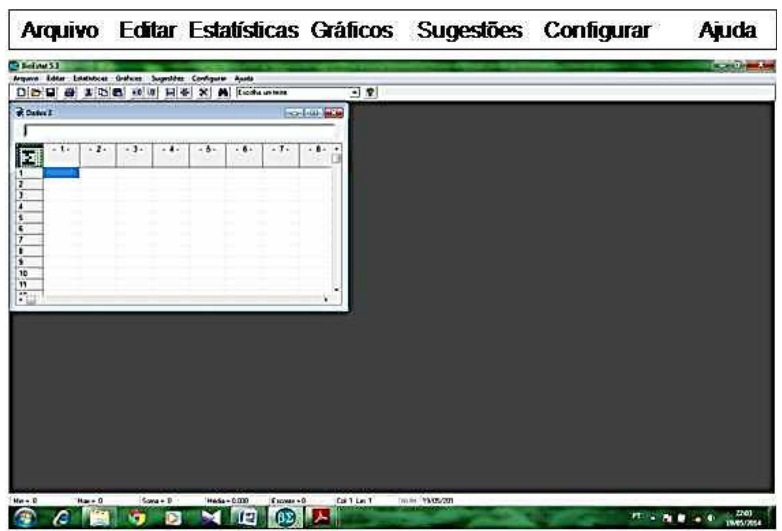

Fonte: Autores (2014).

Além de suas características pedagógicas os softwares foram criados inicialmente para o tratamento de grandes quantidades de informações. A principal meta dos produtores de tecnologia de computadores era desenvolver técnicas e aperfeiçoamento para a minimização de gastos e custos com armazenagem e organização de dados, além, é claro, da diminuição do tempo de processamento (RIBEIRO e DAMASIO, 2006).Neste sentido, devido a qualidade e facilidade apresentada pelo BioEstat, o mesmo passou a ser empregado pelos pesquisadores no tratamento e apresentação de seus dados. Sendo o grande alavanque deste quadro,a quantidade de aplicativos disponibilizados por este (210 aplicativos).

\section{Análise descritiva}

A Análise Descritiva é a fase inicial do processo de estudo dos dados coletados em uma pesquisa, seja ela qualitativa ou quantitativa. Neste tipo de análise, a organizaçãodos dados pode ser realizada de 
três maneiras: por meio de tabelas, de gráficos e de medidas descritivas. Segundo Reis e Reis (2002), utiliza-se de métodos de estatística descritiva para organizar, resumir e descrever os aspectos importantes de um conjunto de características observadas ou comparar tais características entre dois ou mais conjuntos de dados. Os resultados da análise descritiva estão evidenciados na Figura 2, em forma de tabela e gráfico.

Figura 2 - Interface dos resultados obtidos na estatística descritiva e a representação gráfica da distribuição dos valores para cada amostra analisada, através de um boxplot de mediana e quartis.

\begin{tabular}{|c|c|c|c|c|}
\hline 9 & \multicolumn{2}{|c|}{ Estatistica Descritiva } & & - $\square[x$ \\
\hline Arquivo Editar Grófico & & & & \\
\hline & $\begin{array}{c}-1 \\
\text { Fator } \mathrm{A}\end{array}$ & $\begin{array}{c}-2- \\
\text { Fator } B\end{array}$ & $\begin{array}{c}-3- \\
\text { Fator } \mathrm{C}\end{array}$ & $\begin{array}{c}-4- \\
\text { Fator } D\end{array}$ \\
\hline Tamanho da amostra $=$ & 5 & 6 & 7 & 7 \\
\hline Minimo & 15.0000 & 35.0000 & 48.0000 & 62.0000 \\
\hline Máximo & 35.0000 & 50.0000 & 60.0000 & 76.0000 \\
\hline Amplitude Total & 20.0000 & 15.0000 & 12.0000 & 14.0000 \\
\hline Mediana & 25.0000 & 40.0000 & 55.0000 & 70.0000 \\
\hline Primeiro Quantil (25\%) & 20.0000 & 38.5000 & 52.0000 & 66.5000 \\
\hline Terceiro Quartil (75\%) & 30.0000 & 43.7500 & 56.5000 & 72.0000 \\
\hline Desvio Interquartilico & 10.0000 & 5.2500 & 4.5000 & 5.5000 \\
\hline Média Aritmética & 25,0000 & 41.3333 & 54.2857 & 69.2857 \\
\hline Variância & 62.5000 & 28.6667 & 16.5714 & 22.5714 \\
\hline Desvio Padrăo & 7.9057 & 5.3541 & 4.0708 & 4.7509 \\
\hline Erro Padräo & 3.5355 & 2.1858 & 1.5386 & 1.7957 \\
\hline Coeficiente de Variaçäo & $31.62 \%$ & $12.95 \%$ & $7.50 \%$ & $6.86 \%$ \\
\hline Assimetria (g1) & 0.0000 & 0.7905 & -0.1639 & -0.2355 \\
\hline Curtose (g2) & -1.2000 & 0.1906 & -0.4083 & -0.4635 \\
\hline Média Harmónica $=$ & 22.8758 & 40.7860 & 54.0204 & 69.0020 \\
\hline $\mathrm{N}$ (média harmónica) $=$ & 5 & 6 & 7 & 7 \\
\hline Média Geométrica $=$ & 23.9469 & 41.0543 & 54.1536 & 69.1446 \\
\hline $\mathrm{N}$ (média geométrica) $=$ & 5 & 6 & 7 & 7 \\
\hline Variância (geom) $)=$ & 1.0498 & 1.0070 & 1.0025 & 1.0021 \\
\hline Desvio Padrăo (geom.) = & 1.3974 & 1.1349 & 1.0786 & 1.0716 \\
\hline
\end{tabular}

Fonte: Autores (2014).

A obtenção desta análise pelo software BioEstat se mostrou de grande eficácia uma vez que a mesma foi adquirida com um número mínimo de etapas e seus resultados apresentados de forma clara e didática. Estando evidenciado na Tabela 4, seu rendimento qualitativo.

Tabela 4 - Características abordadas durante a execução da análise descritiva e para representação gráfica dos resultados.

\begin{tabular}{lc}
\hline \multicolumn{1}{c}{ Características avaliativas } & $\begin{array}{c}\text { Análise } \\
\text { Descritiva }\end{array}$ \\
\hline Velocidade de execução & Ótima \\
$\begin{array}{l}\text { Apresentação dos resultados } \\
\text { Realização do teste diretamente } \\
\begin{array}{l}\text { Realização do teste } \\
\text { indiretamente }\end{array}\end{array}$ & Sima \\
\hline
\end{tabular}

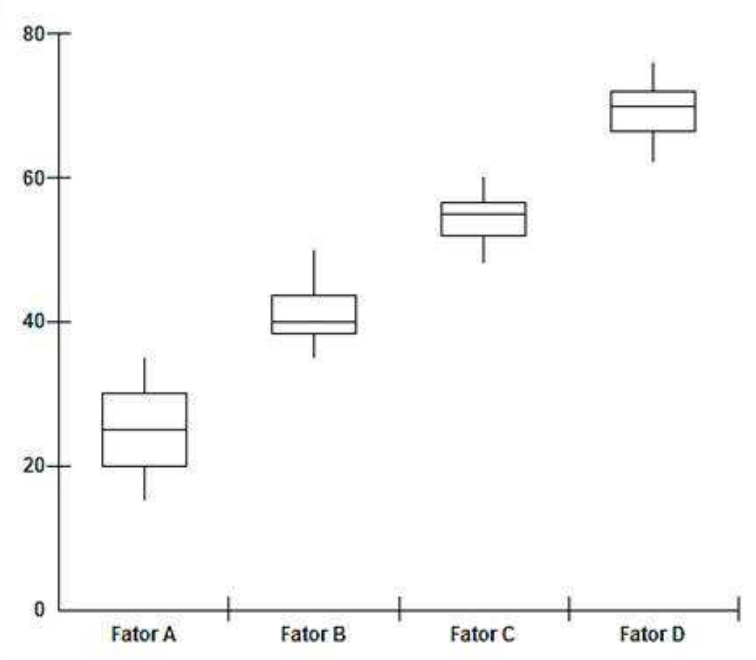

Fonte: Autores (2014).

\section{Testes Paramétricos}

A realização do teste paramétrico com um critério tem por objetivo comparar somente as variações entre os tratamentos, cujo resultado é traduzido no valor do F-teste, complementando-se como exame, a priori(Bonferroni) ou aposteriori(Tukey ou teste $\mathrm{t}$ de Student), dasdiferenças entre as médias amostrais. Os tratamentos podem ter os mesmos números de repetições ou serem desiguais (AYRESet al., 2007).

OBioEstat se mostrou eficaz e de grande praticidade, uma vez que o mesmo o executou-o de forma rápida e clara e com um 
número de etapas diminutas $(<7$ etapas $)$ considerando a importação de dados direto no software. Uma característica do BioEstat para este teste é que o mesmo expõe os resultados das comparações de médias em formas gráficas, facilitando o entendimento e aplicação destes resultados (Figura 3). A linha central refere-se à média do conjunto de dados utilizado nas comparações e as barras em cada coluna referem-se ao desvio padrão observado.

Figura 3 - Interface dos resultados para o teste paramétrico de 1 critério. $<0,01$ significativo e Ns = não significativo ao nível de decisão alfa de 0,01 .

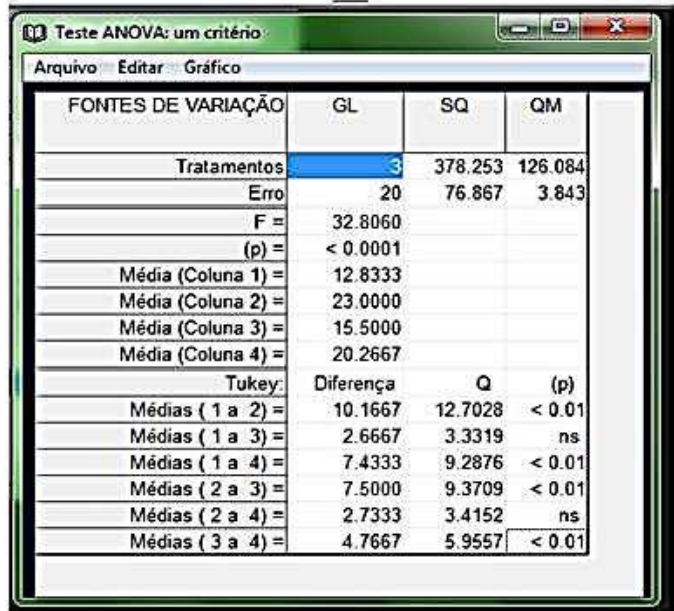

Fonte: Autores (2014).

Para análise de variância com dois critérios foram realizados o teste entre blocos e posteriormente o esquema fatorial (com repetição). A análise em blocos tem por objetivo fazer uma comparação entre os tratamentos verificando a variabilidade entre os blocos (AYRES et al., 2007).O esquema fatorial (com replicação) tem por

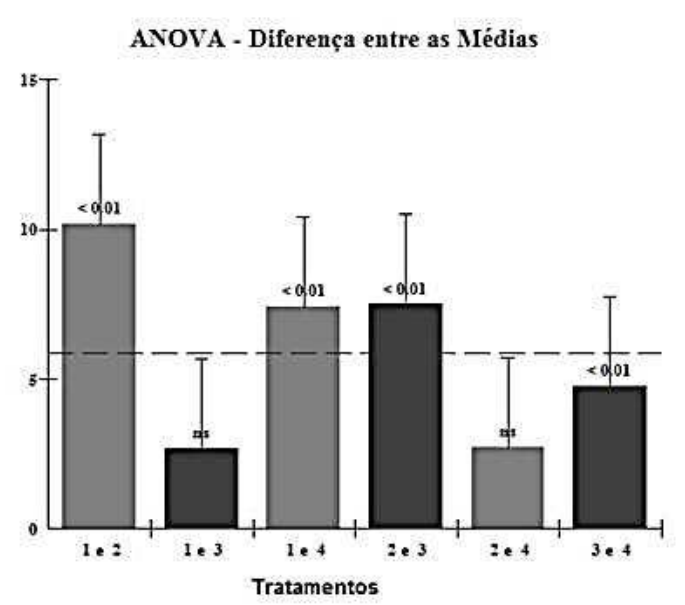

finalidadeaveriguar se há uma possível interação entre os tratamentos e os blocos.

Observou para a análise com dois critérios, que os tratamentos e blocos obtiveramos valores de F-teste e $\mathrm{p}<0,05$ de forma rápida, clara e representada em tabela, o que facilita ainda mais sua compreensão (Figura 4A). 
Figura 4 - Exemplos de exportação de resultados para os testes paramétricos de Dois critérios e Fatorial de dois níveis a x b (com replicação). <0,01 significativo e Ns = não significativo ao nível de decisão alfa de 0,01.

Teste ANOVA: dois critérios

\begin{tabular}{|c|c|c|c|}
\hline FONTES DE VARUGCXO & GL & so & CM \\
\hline Tratamentes & 3 & 378.2533 & 128004 \\
\hline Bioces & 5 & 9900 & 1980 \\
\hline Eno & is & 65.967 & 4.464 \\
\hline F (rratamentos) a & 282419 & & \\
\hline D (trataventos) $=$ & $<0,0001$ & & \\
\hline$F(000(0)$ ) a & 0.4435 & & \\
\hline$D(b l o c 0 s)=$ & 0.8122 & & \\
\hline \multicolumn{4}{|l|}{ Meslas feratsmentos) } \\
\hline Moda (Colona 1) * & 128333 & & \\
\hline Meda (Coluns 2) * & 230000 & & \\
\hline Mods (Coluns 3$)$ ) & is 5000 & & \\
\hline Meda (Coluns 4) " & 20.2667 & & \\
\hline Tukey & 0 & (p) & \\
\hline Nodias $\left(1 a_{2}\right)=$ & 11.7861 & $<001$ & \\
\hline Medias $(103)=$ & 30914 & ms & \\
\hline Modias $(1,04)=$ & 8.6174 & $<001$ & \\
\hline Medias $(2 a 3)=$ & 86947 & $<001$ & \\
\hline Madiss $(204)=$ & 3.1697 & As & \\
\hline Madias $(3,4)=$ & 55259 & $<001$ & \\
\hline
\end{tabular}

Fonte: Autores (2014).

Para o esquema fatorial os valores de F-teste e $\mathrm{p}<0,05$ para os três critérios avaliados (tratamento, blocos e interação entre ambos) estão presentes na Figura 4B. Este apresentou resultado semelhante às análises anteriores para velocidade, apresentação de
(A) ANOVA: fatorial a $\times \mathrm{b}$

(B)

\begin{tabular}{|c|c|c|c|}
\hline FONTES DE VARLCAO & GL & so & OM \\
\hline Tralamentos & 2 & 5289563 & 264.4781 \\
\hline Blocos & 2 & 30.1430 & 15.0715 \\
\hline Interacso & 4 & 21.0170 & 5.2543 \\
\hline Emo & 18 & 7.8267 & 0.4348 \\
\hline$F($ Tratamentos $)=$ & $60825 \times 7$ & $m$ & $m$ \\
\hline Gravs do liberdade = & 2,18 & - & - \\
\hline D(tratamentos) & $<0.0001$ & - & - \\
\hline$F(B i c c 0 s)=$ & 34.6618 & - & - \\
\hline Grous de loerdade * & 2.18 & - & - \\
\hline$P(B \mid<<05)=$ & $<00001$ & - & - \\
\hline$F$ (interaçato) $=$ & 120839 & - & - \\
\hline Graus do leordade = & 4.18 & - & - \\
\hline$P($ interssso) $=$ & 0.0002 & - & - \\
\hline
\end{tabular}

resultados e realização indireta do teste. No entanto, apresentou-se deficiente para a realização do teste diretamente, não aceitando a importação dos dados de outros arquivos, porém, após sua inserção no grid os mesmos podem ser salvos (Tabela 5).

Tabela 5 - Características abordadas durante a execução dos testes paramétricos de 1 critério (inteiramente casualizados), 2 critérios (blocos casualizados) e em esquema fatorial de 2níveis (com replicação).

\begin{tabular}{lccc}
\hline Características avaliativas & \multicolumn{3}{c}{ Testes Paramétricos } \\
\hline & $\begin{array}{c}\text { ANOVA } \\
\text { (1 critério) }\end{array}$ & $\begin{array}{c}\text { ANOVA } \\
(\mathbf{2} \text { critérios })\end{array}$ & $\begin{array}{c}\text { Fatorial } \\
\text { (2 critérios })\end{array}$ \\
\hline Velocidade de execução & Ótima & Ótima & Ótima \\
Apresentação dos resultados & Ótima & Ótima & Ótima \\
Realização do teste diretamente & Sim & Sim & Não \\
Realização do teste indiretamente & Sim & Sim & Sim \\
\hline
\end{tabular}

Fonte: Autores (2014).

\section{Testes Não-paramétricos}

O teste de Friedman consiste em uma avaliaçãonão-paramétrica aplicável ao delineamento em blocos casualizados, para dados mensurados a nível ordinal, abrangendo três ou mais amostras e equivalendo à ANOVA com dois critérios (AYRES, et al., 2007). Nojulgamento de qualidade, osoftware BioEstat se demonstrou eficiente em sua realização, uma vez que os parâmetros de 
agilidade e clareza de resultados se despontaram satisfatórios (Figura 5).

$\mathrm{O}$ teste de Kruskal-Wallis ou teste $\mathrm{H}$ consiste na versão contrária do teste $\mathrm{F}$ na análise paramétrica, o mesmo é utilizado para verificar $\mathrm{o}$ contraste entre $\mathrm{k}$ amostras independentes podendo ter o mesmo número de repetições ou não. Seus valores devem ser a nível ordinal. Neste sentido quando o teste $\mathrm{H}$ for significativo, o BioEstat disponibiliza dois métodos de comparações de médias dos postos: Dunn e Student-Newman Keuls. A análise da qualidade do teste Kruskal-Wallis pelo software BioEstat foi semelhante ao apresentada pelo teste de Friedman, sendo realizado com um número mínimo de etapas (Figura 6).

Para ambos os testes, de Friedman e Kruskal-Wallis, oBioEstat disponibiliza a aquisição dos resultados de comparações de tratamentos (grupos, postos, amostras, etc.) em modelos gráficos, facilitando seu entendimento e aplicação.

Figura 5 - Resultados obtidos do teste não-paramétrico de Friedman. $<0,05$ significativo e Ns = não significativo ao nível de decisão alfa de 0,05 .

Friedman

\begin{tabular}{|c|c|c|c|c|}
\hline & $-1-$ & $.2-$ & $-3-$ & $-4-$ \\
\hline Soma dos Ranks $=$ & 5.0000 & 10.0000 & 15.0000 & 20.0000 \\
\hline Mediana $=$ & 25.0000 & 40.0000 & 55.0000 & 70.0000 \\
\hline Média dos Ranks = & 1.0000 & 2.0000 & 3.0000 & 4.0000 \\
\hline Média dos valores $=$ & 25.0000 & 42.0000 & 53.2000 & 70.4000 \\
\hline Desvio padrã̃o = & 7.9057 & 5.7008 & 3.7014 & 4.2778 \\
\hline Friedman $(F r)=$ & 15.0000 & & & \\
\hline Graus de liberdade $=$ & 3 & & & \\
\hline$(p)=$ & 0.0018 & & & \\
\hline Comparaçöes: & Diferença & (p) & & \\
\hline Ranks 1 e $2=$ & 5 & $\mathrm{~ns}$ & & \\
\hline Ranks 1 e $3=$ & 10 & ns & & \\
\hline Ranks 1 e $4=$ & 15 & $<0.05$ & & \\
\hline Ranks 2 e $3=$ & 5 & $\mathrm{~ns}$ & & \\
\hline Ranks 2 e $4=$ & 10 & ns & & \\
\hline Ranks 3 e $4=$ & 5 & $\mathrm{~ns}$ & & \\
\hline
\end{tabular}

Fonte: Autores (2014).

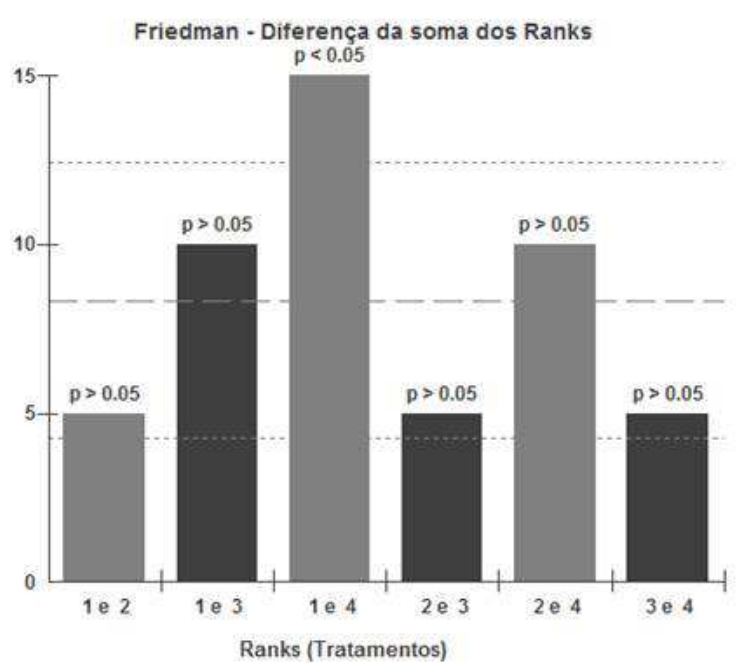


Figura 6 - Resultados obtidos do teste não-paramétrico de Kruskal-Wallis. <0,05 significativo e Ns = não significativo ao nível de decisão alfa de 0,05 . Neste exemplo foi aplicado o teste de Dunn para comparações entre as médias dos postos.

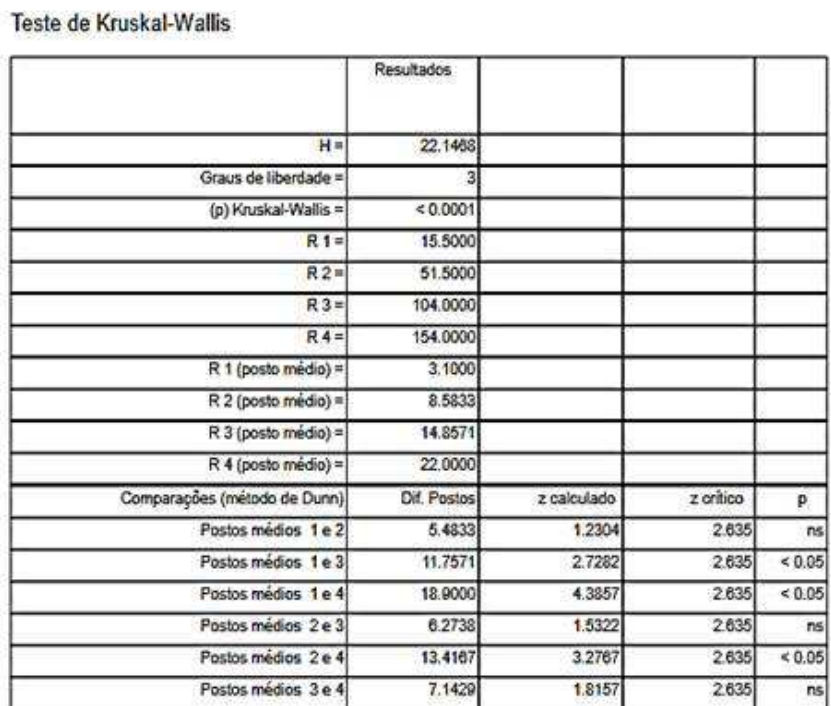

Fonte: Autores (2014).

De forma geral os testes nãoparamétricos de variância se mostraram satisfatórios em termos de agilidade de execução, quantidade mínima de etapas a

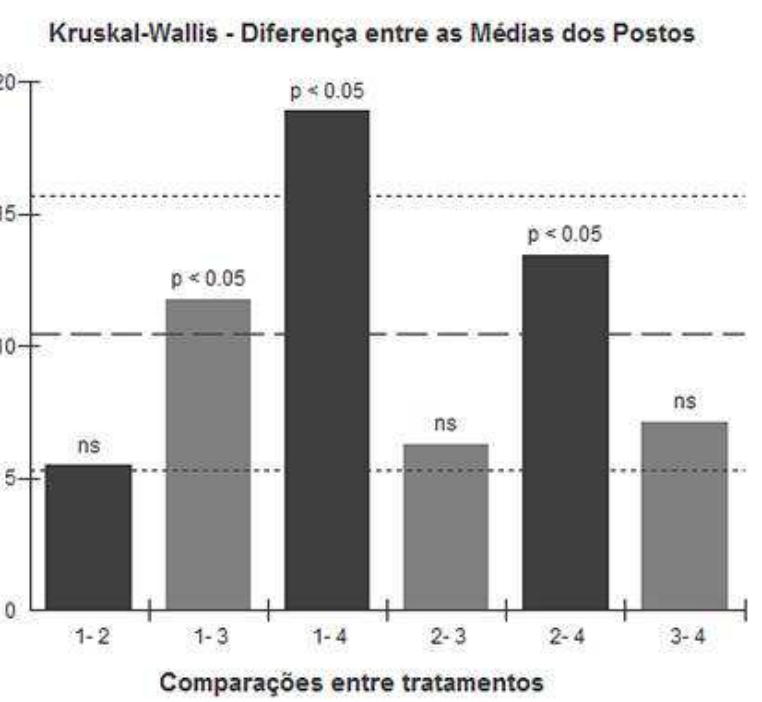

serem concluídas e apresentação dos resultados de forma clara e objetiva pelo software. Outras especificações em relação aos testes estão evidenciadas na Tabela 6.

Tabela 6 - Características abordadas durante a execução dos testes não-paramétricos de Friedman e Kruskal-Wallis no software BioEstat

\begin{tabular}{lcc}
\hline Características avaliativas & \multicolumn{2}{c}{ Teste Não-paramétrico } \\
\hline & Friedman & Kruskal-Wallis \\
\hline Velocidade de execução & Ótima & Ótima \\
Apresentação dos resultados & Ótima & Ótima \\
Realização do teste diretamente & Sim & Sim \\
Realização do teste indiretamente & Sim & Sim \\
\hline
\end{tabular}

Fonte: Autores (2014).

\section{CONCLUSÃO}

O software BioEstat se mostrou eficaz como auxílio as disciplinas de Estatísticas na graduação, devido sua praticidadee aplicabilidade em diversas áreas.Apresentando potencialidade de despertar a atenção e o interesse do aluno para com a estatística.

\section{REFERÊNCIAS BIBLIOGRÁFICAS}

AYRES, M., AYRES Jr, M., AYRES, D. L., SANTOS, A. A. S. Bioestat 5.0 aplicações estatísticas nas áreas das ciências biológicas e médicas. Belém: IDSM, 2007.364p. 
CAMPOS, G. H. B.; CAMPOS, F. C. A. Qualidade de Software Educacional. In: CAMPOS, G. H. B.; CAMPOS, F. C. A. Qualidade de software: Teoria e Prática. Campinas: Makron, 2001.

COEDANI, L. K. O Ensino de Estatística na Universidade e a controvérsia sobre os fundamentos da inferência. Tese de doutorado. USP, São Paulo, 2001.

FREIRE, P.:Pedagogia da autonomia: saberes necessários à prática educativa. São Paulo: Paz e Terra, 1996.

ISO/CD8402, 1990, Quality Concepts and Terminology - Part One: Generic Terms and Definition, International Standards Organization.

Milani,E. (2001). A informática e a comunição matemática. In: K.S. Smole\& M.L. Diniz (Orgs.); Ler, escrever e resolver problemas: Habilidades básicas para aprender matemática (pp.176-200). Porto Alegre: Artmed.

RIBEIRO, C. E. N.; DAMASIO, E. Software livre para bibliotecas, sua importância e utilização: o caso GNUTECA. Revista Digital de Biblioteconomia e Ciência da Informação, Campinas, v.4, n. 1, p. 70-86, jul./dez. 2006 .

REIS, E. A.; REIS, I. A. Análise descritiva de dados Síntese numérica. Relatório Técnico RTP - 02/2002. Série Ensino. 